\title{
Characterization of the domain chaos convection state by the largest Lyapunov exponent
}

\author{
A. Jayaraman, ${ }^{1}$ J. D. Scheel, ${ }^{2}$ H. S. Greenside, ${ }^{1, *}$ and P. F. Fischer ${ }^{3}$ \\ ${ }^{1}$ Department of Physics, Duke University, Durham, North Carolina 27708, USA \\ ${ }^{2}$ Department of Physics, California Institute of Technology 114-36, Pasadena, California 91125, USA \\ ${ }^{3}$ Mathematics and Computer Science Division, Argonne National Laboratory, Argonne, Illinois 60439, USA
}

(Received 9 September 2005; published 26 July 2006)

\begin{abstract}
Using numerical integrations of the Boussinesq equations in rotating cylindrical domains with realistic boundary conditions, we have computed the value of the largest Lyapunov exponent $\lambda_{1}$ for a variety of aspect ratios and driving strengths. We study in particular the domain chaos state, which bifurcates supercritically from the conducting fluid state and involves extended propagating fronts as well as point defects. We compare our results with those from Egolf et al., [Nature 404, 733 (2000)], who suggested that the value of $\lambda_{1}$ for the spiral defect chaos state of a convecting fluid was determined primarily by bursts of instability arising from short-lived, spatially localized dislocation nucleation events. We also show that the quantity $\lambda_{1}$ is not intensive for aspect ratios $\Gamma$ over the range $20<\Gamma<40$ and that the scaling exponent of $\lambda_{1}$ near onset is consistent with the value predicted by the amplitude equation formalism.
\end{abstract}

DOI: 10.1103/PhysRevE.74.016209

PACS number(s): 47.54.-r, 47.20.Bp, 47.20.Ky, 47.52.+j

\section{INTRODUCTION}

Spatiotemporal chaotic states remain poorly understood despite their widespread occurrence in experimental sustained nonequilibrium systems and in mathematical models of such systems [1-3]. Researchers do not know how to predict the parameter values for which spatiotemporal chaos occurs, the way a spatiotemporal chaotic state changes as some parameter is varied, and the values of physically significant quantities such as transport coefficients of matter and energy [4]. Also poorly understood is how the chaotic temporal behavior is related to the spatial disorder and whether knowledge of, say, the spatial structure may suffice to make predictions about the temporal dynamics.

In a recent paper [5], Egolf et al. used numerical simulations to study some of these questions in the context of the spiral defect chaos (SDC) state of an incompressible convecting fluid. Spiral defect chaos is one of the more thoroughly investigated spatiotemporal chaotic states by experimentalists [6-12] and has also been studied by many theorists [13-20]. One of the several interesting results reported in the Egolf et al. paper was that the value of the largest Lyapunov exponent $\lambda_{1}$ seemed to be determined mainly by short-lived, spatially localized dislocation nucleation events. This observation suggests a quantitative connection between the strength of the chaotic behavior as measured by $\lambda_{1}$ and certain isolated spatial features of the fluid flow.

In this paper, we investigate the generality of this result by using numerical simulations to study the domain chaos state of an incompressible rotating convecting fluid with experimentally realistic boundary conditions. In contrast, the simulations by Egolf et al. studied the SDC case and used periodic boundary conditions. We do not find a simple relation between defect statistics and $\lambda_{1}$, contrary to the SDC results of Egolf et al.

\footnotetext{
*Author to whom correspondence should be addressed; Electronic address: hsg@phy.duke.edu
}

Domain chaos is a spatiotemporal chaotic state that has also been studied by experimentalists [21-25] and by theorists [26-31] and that has two features of special interest. First, domain chaos is one of the few experimentally known spatiotemporal chaotic states that bifurcates supercritically from a time-independent, spatially uniform state (here the motionless conducting state of the fluid). Supercritical bifurcations to chaos from simple spatial states provide important opportunities for theoretical progress because controlled expansions can be performed in the small parameter $\epsilon$ that measures the closeness to the onset of instability of the uniform state. Because the bifurcation to domain chaos is supercritical, we further expect that as $\epsilon \rightarrow 0^{+}$, certain quantities that characterize the temporal and spatial properties of the domain chaos state should scale as power laws in $\epsilon$ with corresponding critical exponents $\alpha$. Possible relations between the temporal and spatial properties of a spatiotemporal chaotic state can then be tested quantitatively by comparing their critical exponents. Critical exponents are especially interesting to determine for dynamical invariants such as the largest Lyapunov exponent $\lambda_{1}[5,32-36]$ and the Lyapunov fractal dimension $D_{\mathcal{L}}[3,5,37-40]$ because the critical exponents observed experimentally for the correlation time and correlation length (derived from two-point correlation functions) do not agree with the values predicted by the amplitude equation formalism $[21,26,27,30,31]$. This discrepancy is arguably one of the more important problems to understand in nonequilibrium physics because there are so few quantitative theoretical predictions concerning spatiotemporal chaos.

A second useful feature of domain chaos is that at least two kinds of topological defects occur in the convection roll pattern: localized defects consisting of dislocations or clusters of dislocations, and extended defects in the form of propagating fronts that separate a patch of rolls with one orientation from a neighboring patch of rolls with a different orientation. We note that although there are extended spirals in spiral defect chaos, these can be considered as driven by the interaction of two point defects, a focus and a dislocation, and so should not act as an extended defect [14]. The 
presence of extended defects in domain chaos raises the possibility that the strength of the chaos as defined by the exponent $\lambda_{1}$ may not be understood simply in terms of defect events because the dynamics no longer consists of spatially and temporally localized fluid structures.

We also summarize calculations that explore how the exponent $\lambda_{1}$ scales near the onset of convection and how it varies with the aspect ratio $\Gamma$. We show in Sec. III B that the exponent $\lambda_{1}$ varies linearly with $\epsilon$, a result that is consistent with the amplitude equation formalism. In Sec. III C, we also show that the exponent $\lambda_{1}$ does not act as an intensive quantity because it increases with $\Gamma$ over the range $20 \leqslant \Gamma \leqslant 40$. This result is surprising because general arguments [3] suggest that $\lambda_{1}$ should be intensive and hence independent of the shape or size of a sufficiently large nonequilibrium system.

The rest of this paper is organized as follows. In Sec. II, we define the largest Lyapunov exponent $\lambda_{1}$ and the instantaneous Lyapunov exponent $\lambda_{1}^{\text {inst }}$ and provide some details about our numerical methods. In Sec. III, we discuss our results: we compare spatial structure with $\lambda_{1}^{\text {inst }}$ and examine how $\lambda_{1}$ scales near onset and varies with aspect ratio. In Sec. IV, we summarize the important points and relate them to recent work.

\section{METHODS}

In this section, we discuss the definitions, equations, and numerical algorithms used to obtain the results discussed in Sec. III.

\section{A. The instantaneous largest Lyapunov exponent $\lambda_{1}^{\text {inst }}$ and the largest Lyapunov exponent $\lambda_{1}$}

Chaotic behavior is often characterized by dynamical invariants [41] because these quantities in principle do not depend on the choice of observable. Two dynamical invariants, the largest Lyapunov exponent $\lambda_{1}$ and the Lyapunov fractal dimension $D_{\mathcal{L}}$, have been especially used to characterize the temporal dynamics of a spatiotemporal chaotic state. We discuss these briefly in turn.

The largest Lyapunov exponent $\lambda_{1}$ is the dynamical invariant most easily and accurately estimated from experimental time series [42], and its inverse $\lambda_{1}^{-1}$ is widely identified as the time scale beyond which accurate predictions of a chaotic system cannot be made. We note that there are subtleties regarding the physical interpretation and relevance of the exponent $\lambda_{1}$ for spatially extended systems, since these large systems have many positive Lyapunov exponents so that there is no longer a distinguished time scale of instability. One also must keep in mind that the Lyapunov exponents characterize the growth of infinitesimal perturbations, while physical systems are often subject to perturbations of a finite and perhaps sizable magnitude [43].

The Lyapunov fractal dimension $D_{\mathcal{L}}$ basically counts the number of active degrees of freedom and hence is a useful measure of dynamical complexity [41]. (It has been conjectured for some systems to be the same as the information dimension $D_{1}$.) This dimension $D_{\mathcal{L}}$ is important for spatiotemporal chaos because it is the only fractal dimension whose value can be estimated for high-dimensional, spatially extended dynamical systems. Even then, this is possible only if evolution equations and their linearization about some chaotic orbit are explicitly known [44]. It is still not known how to estimate the quantity $D_{\mathcal{L}}$ from time series [45], so its properties for experimental systems are known only indirectly by numerical integrations of quantitatively accurate evolution equations. Because of the great computational expense to compute $D_{\mathcal{L}}$, in this paper we study only the exponent $\lambda_{1}$ and its instantaneous version $\lambda_{1}^{\text {inst }}$.

For a flow $d \mathbf{y} / d t=\mathbf{F}(\mathbf{y})$ where $\mathbf{y}$ and $\mathbf{F}$ are $N$-dimensional vectors, the largest Lyapunov exponent $\lambda_{1}$ is defined by the following limit [44]:

$$
\lambda_{1}=\lim _{t \rightarrow \infty} \frac{1}{t} \ln \left(\frac{\|\delta \mathbf{y}(t)\|}{\|\delta \mathbf{y}(0)\|}\right),
$$

provided that this limit exists. Here the $N$-dimensional vector $\delta \mathbf{y}(t)$ satisfies the linearized evolution equation

$$
\frac{d \delta \mathbf{y}}{d t}=\mathbf{J}(\mathbf{y}(t)) \cdot \delta \mathbf{y}
$$

with Jacobian $\mathbf{J}=\delta \mathbf{F} / \delta \mathbf{y}$ and initial value $\delta \mathbf{y}(0)$. The orbit $\mathbf{y}(t)$ defines some attractor of interest.

To study how $\lambda_{1}$ varies over short intervals of time, we define an instantaneous largest Lyapunov exponent $\lambda_{1}^{\text {inst }}(t)$ by [46]

$$
\lambda_{1}^{\text {inst }}(t)=\frac{d}{d t} \ln \left(\frac{\|\delta \mathbf{y}(t)\|}{\|\delta \mathbf{y}(0)\|}\right) .
$$

For numerical integrations of the flow and of its linearization Eq. (2), the derivative in Eq. (3) can be approximated by a finite difference over a time interval $\Delta T$ that is usually somewhat larger than the constant integration step $\Delta t$, so that $\lambda_{1}^{\text {inst }}(t)$ measures the rate at which two nearby states diverge over the times $t$ and $t+\Delta T$. The average of $\lambda_{1}^{\text {inst }}(t)$ over all time gives the largest Lyapunov exponent $\lambda_{1}$.

The above definition of $\lambda_{1}^{\text {inst }}$ allows us to study short time contributions to the Lyapunov exponent. One can, however, define other quantities that also provide related short-time information. Since in chaotic systems $\|\delta \mathbf{y}(t)\|$ grows on average exponentially with a rate $\lambda_{1}$, a local exponent $\lambda_{1}^{\text {loc }}$ can be defined as

$$
\lambda_{1}^{\operatorname{loc}}(t)=\ln \left(\frac{\|\delta \mathbf{y}(t)\|}{\|\delta \mathbf{y}(0)\|}\right)-\lambda_{1} t
$$

This quantity shows the local deviations from the average exponential rate. Note that $\lambda_{1}$ needs to be computed by a separate calculation first. The three quantities are related in the following manner:

$$
\lambda_{1}^{\text {inst }}(t)=\lambda_{1}+\frac{d}{d t} \lambda_{1}^{\text {loc }}(t)
$$

We will define one more quantity, one that is more fundamental than the others: 


$$
S_{\lambda}=\ln \left(\frac{\|\delta \mathbf{y}(t)\|}{\|\delta \mathbf{y}(0)\|}\right) .
$$

The other Lyapunov diagnostic quantities in Eqs. (1), (3), and (4) can be found from (6).

The quantities in Eqs. (3), (4), and (6) will fluctuate along a given chaotic orbit. For small-dimensional systems, say $D<5$, researchers have studied how short-time exponents vary along the orbit $[47,48]$, how the distribution of the exponents can be used to characterize the orbit [49], and how the distributions converge to the infinite-time limit [50]. However, it remains poorly understood which details of these papers extend to high-dimensional spatially extended systems (for example, the spiral defect chaos state in a periodic domain of aspect ratio 64 was estimated to have a Lyapunov fractal dimension of 80 [5]).

To gain some intuition about the instantaneous largest exponent $\lambda_{1}^{\text {inst }}$ and the local exponent $\lambda_{1}^{\text {loc }}$ in a simpler context than the Boussinesq equations, we show their behavior for a low-dimensional chaotic attractor of the Lorenz equations

$$
\dot{x}=\sigma(y-x), \quad \dot{y}=r x-y-x z, \quad \dot{z}=-b z+x y,
$$

for the parameter values $\sigma=16, b=4$, and $r=45.92$. (These values were chosen to agree with those used in Ref. [51], for which other properties of this chaotic attractor have been calculated.) We integrated Eqs. (7) and their linearizations about the chaotic orbit using a standard fourth-order RungeKutta scheme with constant time step $\Delta t=10^{-3}$ and used Eqs. (1), (3), and (4) to estimate $\lambda_{1}, \lambda_{1}^{\text {inst }}$, and $\lambda^{\text {loc }}$, respectively. A time interval of $\Delta T=4 \Delta t$ was used when approximating Eq. (3) with a finite difference. Figure 1(a) shows how the quantity $\lambda_{1}$ converges noisily to its infinite time value $\lambda_{1} \approx 1.50$ as the integration time $T$ increases, while Figure 1(b) shows how the instantaneous exponent $\lambda_{1}^{\text {inst }}$ varies over the same time range. The dynamics is not uniformly chaotic, and local values can deviate substantially from the mean value $\lambda_{1}$ $\approx 1.50$. Figure 2 shows the relationship between $\lambda_{1}^{\text {inst }}$ (dotted line) and $\lambda_{1}^{\text {loc }}$ (solid line). Note that the regions where $\lambda_{1}^{\text {loc }}$ is increasing are correlated with the regions where $\lambda_{1}^{\text {inst }}$ is positive, as is expected because $\lambda_{1}^{\text {inst }}$ is related to the derivative of $\lambda_{1}^{\text {loc }}$ as in Eq. (5).

In this paper, we will not use $\lambda_{1}^{\text {loc }}$ for the analysis because $\lambda_{1}^{\text {inst }}$ directly allows us to pinpoint the events that provide a maximal contribution to $\lambda_{1}$. The quantity $\lambda_{1}^{\text {inst }}$ also has a practical advantage in that it can be computed for short intervals even without the knowledge of the long time average $\lambda_{1}$.

We note, however, that the value of a high peak in $\lambda_{1}^{\text {inst }}$ is not by itself necessarily significant because it is the area under the peak that contributes toward $\lambda_{1}$. Hence, significant dynamical events may be more apparent in the plot of the quantity $S_{\lambda}$ defined in (6). The quantities $\lambda_{1}^{\text {inst }}$ and $S_{\lambda}$ are compared in Fig. 6.

For the Lorenz equations, local maxima of $\lambda_{1}^{\text {inst }}$ correspond to specific regions in phase space, as indicated by the thick dots in Fig. 3. These are the most unstable regions of this attractor. It is precisely this kind of relationship between phase space dynamics and strength of the chaos that we seek for the rotating convection system.
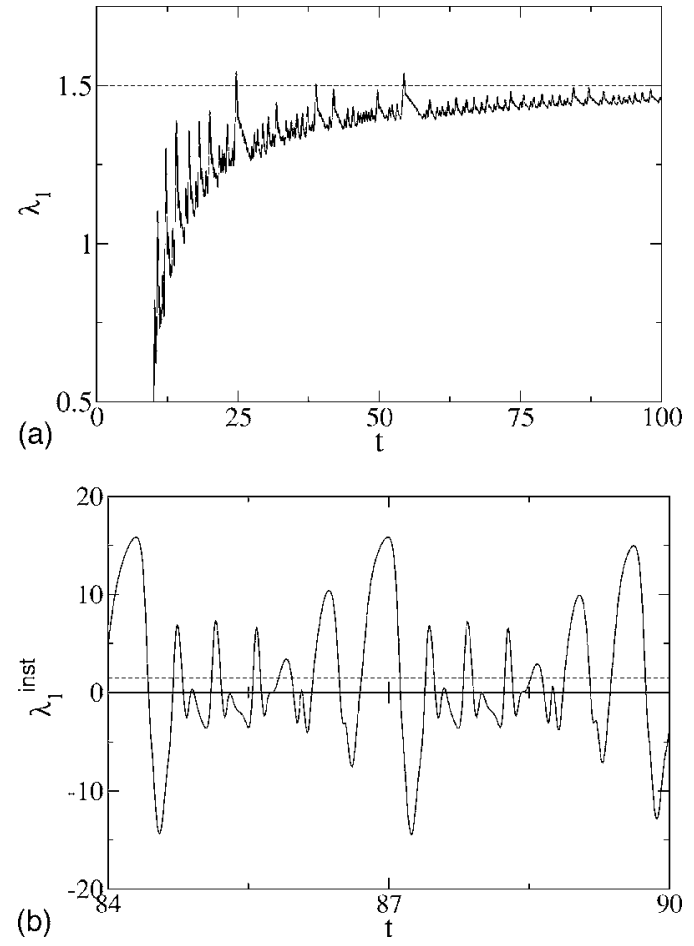

FIG. 1. (a) Convergence of the largest Lyapunov exponent, Eq. (1), to its asymptotic value $\lambda_{1} \approx 1.5$ for a chaotic attractor of the Lorenz equations, Eq. (7), for parameters $\sigma=16, b=4$, and $r$ $=45.92$. (b) The evolution of the instantaneous largest Lyapunov exponent $\lambda_{1}^{\text {inst }}$, Eq. (3), for the same chaotic attractor. The chaos varies substantially in strength over the orbit. The dashed line indicates the asymptotic value of $\lambda_{1} \approx 1.5$.

\section{B. Numerical integration of the 3D Boussinesq equations with rotation}

In this section, we briefly describe how we used a parallel spectral element method to integrate the three-dimensional Boussinesq equations in large cylindrical domains with boundary conditions close to those of actual experiments [21].

Spatiotemporal chaos is perhaps most cleanly studied in large, homogeneous cells (to reduce the influence of lateral walls and to approximate the translation invariance that sim-

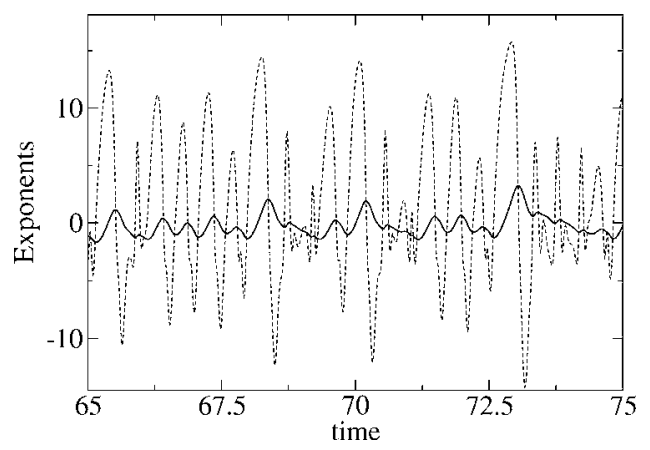

FIG. 2. The instantaneous Lyapunov $\lambda_{1}^{\text {inst }}$ (dotted line) is plotted along with the local Lyapunov exponent $\lambda_{1}^{\text {loc }}$ (solid line) for the Lorenz Eqs. (7) for the parameter values $\sigma=16, b=4$, and $r$ $=45.92$. 


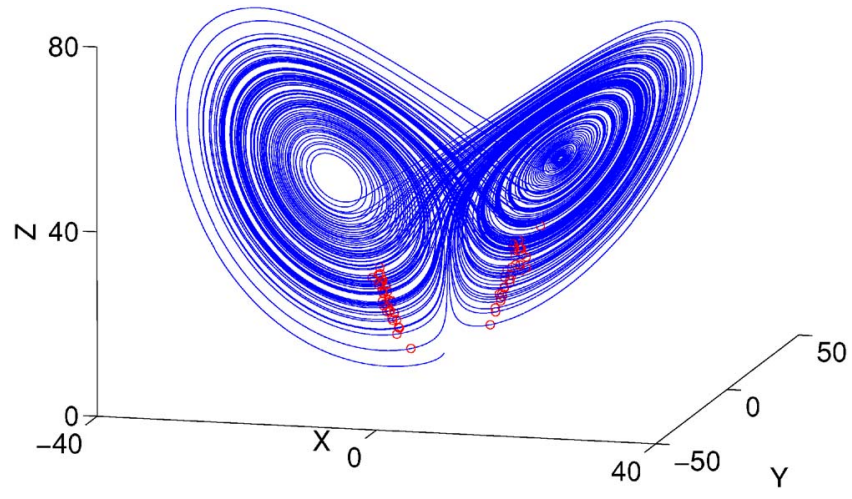

FIG. 3. (Color online) The Lorenz attractor is shown for Eqs. (7) with the parameters $\sigma=16, b=4$, and $r=45.92$. The most chaotic region of the attractor, where the quantity $\lambda_{1}^{\text {inst }}$ has a local maximum larger than $5 \lambda_{1}$, are indicated by the cluster of dots.

plifies theory), over large times (to obtain nontransient behavior and good statistics), and close to onset (so that analytical progress may be possible). This is a difficult regime to simulate a nonequilibrium system quantitatively because many spatial modes are then needed to represent the spatial fields, many integration steps are needed to explore long times, and critical slowing means that even more time steps are needed.

The Boussinesq equations in a rotating Rayleigh-Bénard convection cell are

$$
\frac{\partial \mathbf{u}}{\partial t}+\mathbf{u} \cdot \nabla \mathbf{u}=-\nabla p+\sigma \nabla^{2} \mathbf{u}+R \sigma\left(T-T_{0}\right) \hat{\mathbf{z}}-2 \sigma \Omega \hat{\mathbf{z}} \times \mathbf{u},
$$

$$
\begin{gathered}
\frac{\partial T}{\partial t}+\mathbf{u} \cdot \nabla T=\nabla^{2} T, \\
\boldsymbol{\nabla} \cdot \mathbf{u}=0 .
\end{gathered}
$$

Here $\mathbf{u}(t, x, y, z)=\left(u_{x}, u_{y}, u_{z}\right)$ is the fluid velocity field, $T(t, x, y, z)$ is the fluid temperature field, and $p(t, x, y, z)$ is the fluid pressure field. The $x$ and $y$ coordinates lie in the horizontal plane, and $z$ lies in the vertical direction, with the gravitational acceleration in the $-\hat{\mathbf{z}}$ direction. The field $T_{0}(z)$ is the linearly conducting temperature profile in the absence of convection $(\mathbf{u}=\mathbf{0})$. The equations and parameters have been nondimensionalized in the usual way [52] by using the cell height $d$ as the length scale; the thermal vertical diffusion time $\tau_{v}=d^{2} / \kappa$ as the time scale, where $\kappa$ is the fluid thermal diffusivity; and $\Delta T$, the temperature difference between the top and bottom plate, as the temperature scale. The parameters then are (i) the Prandtl number $\sigma=\nu / \kappa$, where $\nu$ is the kinematic viscosity, (ii) the Rayleigh number $R$ $=\alpha g \Delta T d^{3} / \kappa \nu$, where $\alpha$ is the thermal expansion coefficient and $g$ is the acceleration of gravity, and (iii) the angular velocity $\Omega$ in the $\hat{\mathbf{z}}$ direction (the cylindrical cells are horizontal and rotate about their centers). We use the reduced Rayleigh number
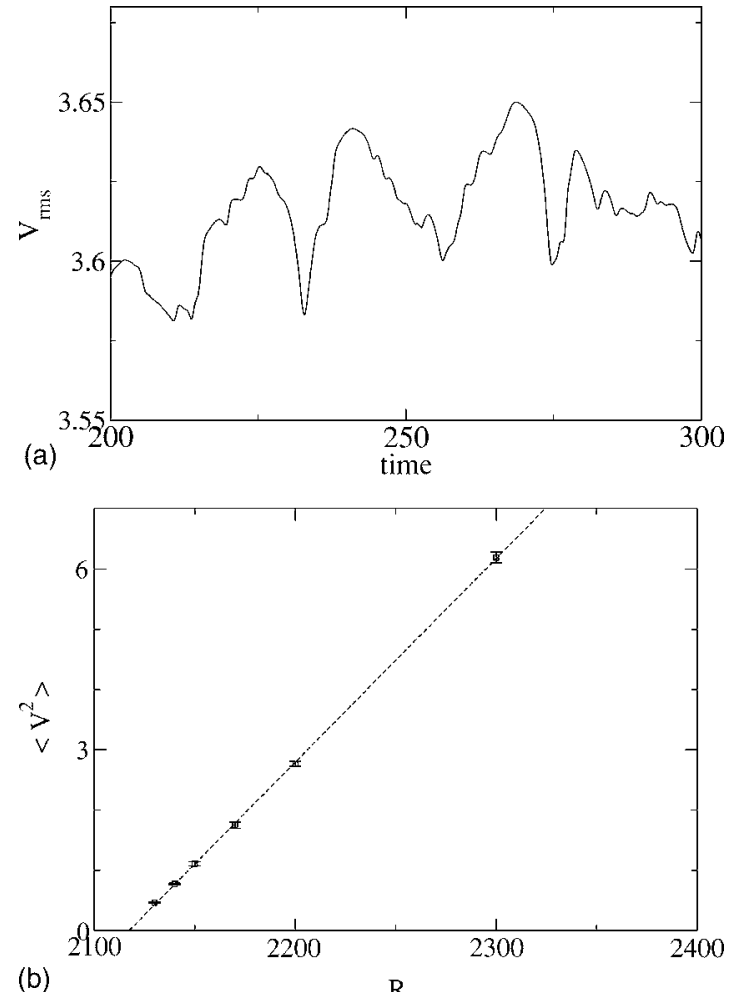

FIG. 4. (a) The evolution of the root-mean-square velocity $V_{\text {rms }}$ of a domain chaos state in a cylinder of aspect ratio 20 for parameters $R=2500, \sigma=0.93$, and $\Omega=15$. (b) Mean square velocity $\left\langle V^{2}\right\rangle$ versus $R$ for the same parameters as in panel (a). The dotted line shows a best fit of the data, which yielded the value $R_{c} \approx 2117$. Linear stability theory for an infinite aspect ratio rotating cell predicts the onset value $R_{c}=2110.5$.

$$
\epsilon=\frac{R-R_{c}(\Gamma)}{R_{c}(\Gamma)}
$$

to denote the closeness to the onset of convection, where $R_{c}(\Gamma)$ is the empirical onset of convection in a finite aspect ratio system, as determined by extrapolating the amplitudes of the nonlinear states just above onset to zero amplitude (see Fig. 4). For convection, $R_{c}(\Gamma)-R_{c}(\infty) \propto \Gamma^{-2}$ for $\Gamma>5$, so the finite aspect ratio value $R_{c}(\Gamma)$ approaches the infinite aspect ratio value rather rapidly.

The Boussinesq equations were solved with no-slip boundary conditions $\mathbf{u}=\mathbf{0}$ on all walls of a cylindrical domain of aspect ratio $\Gamma=L / d$, where $L$ is the radius of the cylinder. The temperature field $T$ took on specified constant values on the bottom and top plates $[T(z=0)=1$ and $T(z$ $=1)=0]$, and we used conducting lateral boundary conditions $T=T_{0}(z)$ on the sidewalls, to approximate reasonably well the experimental lateral conditions [53]. No boundary condition was needed for the pressure $p$ because that field does not satisfy a dynamical equation but instead is a Lagrange multiplier that adjusts its value instantly at each time step so that the fluid remains incompressible.

The simulations of the full Boussinesq equations and the corresponding linearized equations were carried out by using an optimized parallel spectral element code called nek5000, 
which was developed by Tufo and Fischer [54]. The spectral element method provides an efficient high-order representation of spatial differential operators and can treat computational domains with complex geometric shapes and almost arbitrary boundary conditions with high accuracy. In the convection simulations discussed below, each computational domain was divided into topologically cubic elements of approximately equal size and unit volume (where the unit of length is the depth of the fluid). Within each element, the velocity, pressure, and temperature fields and their derivatives were represented as tensor products of 10th- or 12thorder polynomials, corresponding to about 11 to 13 mesh points per half-roll in each direction. The integrations were carried out on a variety of parallel computers and used from 32 to 512 processors at a time. The aspect ratio 20 cells took typically 80 hours on 128 nodes of the Blue Horizon machine at SDSC to span one horizontal diffusion time $\Gamma^{2}$.

A second-order semi-implicit time-integration scheme was used to evolve the solutions $[55,56]$. A constant time step $\Delta t$ was chosen for each calculation that was just smaller than the largest value consistent with the Courant-FriedrichsLevy (CFL) condition for numerical stability of the advection terms (which are advanced explicitly and so determine the smallest upper bound on the step size). The constant time step had different values for different choices of Rayleigh number $R$ and angular speed $\Omega$ and were typically in the range of 0.001-0.005 vertical thermal diffusion times, so 50000 or more total time steps were needed per simulation to study dynamics of order one horizontal diffusion time or longer. The fact that the largest time step is bounded by a CFL condition that depends on the magnitude of the velocity field but not on the time scale of the dynamics (e.g., the CFL condition holds even for time-independent convection) is a well-known weakness of semi-implicit time-integration algorithms for fluid codes. Despite a recent effort to find more efficient algorithms that choose a larger time step consistent with the physical time scale [57], semi-implicit integration schemes remain the most efficient choice for the spectralelement method.

This code has been used in several recent calculations $[4,20,31,56,58-60]$ and has been validated in several ways for a range of spatial and temporal resolutions and for a range of physical parameters. As one example, for the spacetime resolutions used in this paper and for the parameter values $\Gamma=20, \sigma=0.93$, and $\Omega=15$, we were able to reproduce to within $0.3 \%$ the critical Rayleigh number $R_{c}(\Omega)$ $=2110.5$ predicted by linear stability theory [61] in an infinite aspect ratio rotating cell, by extrapolating the RMS velocity of nontransient domain chaos states near onset to zero velocity in a $\Gamma=20$ cell (see Fig. 4). The fact that our extrapolated value $R_{c} \approx 2117$ is slightly larger than the theoretical result is likely a consequence of the finite aspect ratio rather than insufficient space-time resolutions, since the error becomes smaller with increasing aspect ratio for a fixed spatial resolution per unit length.

\section{Calculation of the exponents $\lambda_{1}$ and $\lambda_{1}^{\text {inst }}$}

In order to compute the largest Lyapunov exponent $\lambda_{1}$ and the instantaneous exponent $\lambda_{1}^{\text {inst }}$, the Boussinesq equations
(8)-(10) and their versions linearized about a given orbit were integrated with a constant time step as described above. The perturbation vector $\delta \mathbf{y}=(\delta \mathbf{u}, \delta T)$, consisting of the three components of the linearized, nondimensional velocity field and of the linearized, nondimensional temperature field, was then used in the definitions (1) and (3) to estimate the values of the exponents $\lambda_{1}$ and $\lambda_{1}^{\text {inst }}(t)$. One of two Euclidean norms over the interior volume $V$ was used in the definitions:

$$
\begin{gathered}
\|\delta \mathbf{y}\|=\sqrt{\frac{1}{V} \int_{V}\left(\delta \mathbf{u}^{2}+\delta T^{2}\right) d V}, \\
\|\delta \mathbf{y}\|=\sqrt{\frac{1}{V} \int_{V}\left(R_{c} \delta \mathbf{u}^{2}+\sigma R^{2} \delta T^{2}\right) d V .}
\end{gathered}
$$

The first norm is conventional, and the second is the one used by Cross [62] in his analytical work on the Boussinesq equations. The latter has the advantage that the temperature perturbation term $\sigma R^{2} \delta T^{2}$ is then of the same order of magnitude as the velocity perturbation term $R_{c} \delta \mathbf{u}^{2}$. Both norms eventually converge to the same $\lambda_{1}$, but the second norm tends to converge faster.

Since the perturbation field is expected to grow exponentially fast in a chaotic system, we rescale its norm to unity every 20 time steps (typical time steps are from $0.001-0.005$ vertical diffusion times) in order to keep the derivatives of various fields of order unity. Since the evolution equation for the perturbation is linear, this rescaling does not affect the dynamics. Serendipitously, the running sum of the logarithm of the norm used to rescale the perturbation gives us $S_{\lambda}$, (6), which can then be used to compute $\lambda_{1}$ and $\lambda_{1}^{\text {inst }}$ from Eqs. (1) and (3), respectively.

The calculations of the exponents $\lambda_{1}$ and $\lambda_{1}^{\text {inst }}$ were validated in several ways. For example, simulations of a nonchaotic convecting flow confirmed that both exponents converged to a negative value for the leading Lyapunov exponent. The value of the Lyapunov exponent so obtained was explicitly checked by performing two full nonlinear simulations with slightly differing initial conditions. The difference in the velocity and temperature fields from those two simulations was found to decay exponentially with an exponent close to that of the leading Lyapunov exponent. Such a calculation was also repeated for the chaotic case. Results from the linearized code matched those obtained from two nonlinear codes with slightly differing initial conditions. We also repeated the calculations of Egolf et al. [5] on a periodic cell of aspect ratio $\Gamma=64$ with $\Omega=0, \sigma=1, N=4096$ spectral elements, and time step $\Delta t=2 \times 10^{-3}$. Figure 5(a) shows the evolution of $\lambda_{1}^{\text {inst }}$ using our code for the SDC state. The results agree qualitatively with the results of Egolf $e t$ al. [63]. This is a strong confirmation of the correctness of our code, since our code is significantly different from the Egolf et al. spectral code.

\section{RESULTS AND DISCUSSION}

In this section, we discuss the relation between the instantaneous largest Lyapunov exponent $\lambda_{1}^{\text {inst }}$ and the spatial struc- 

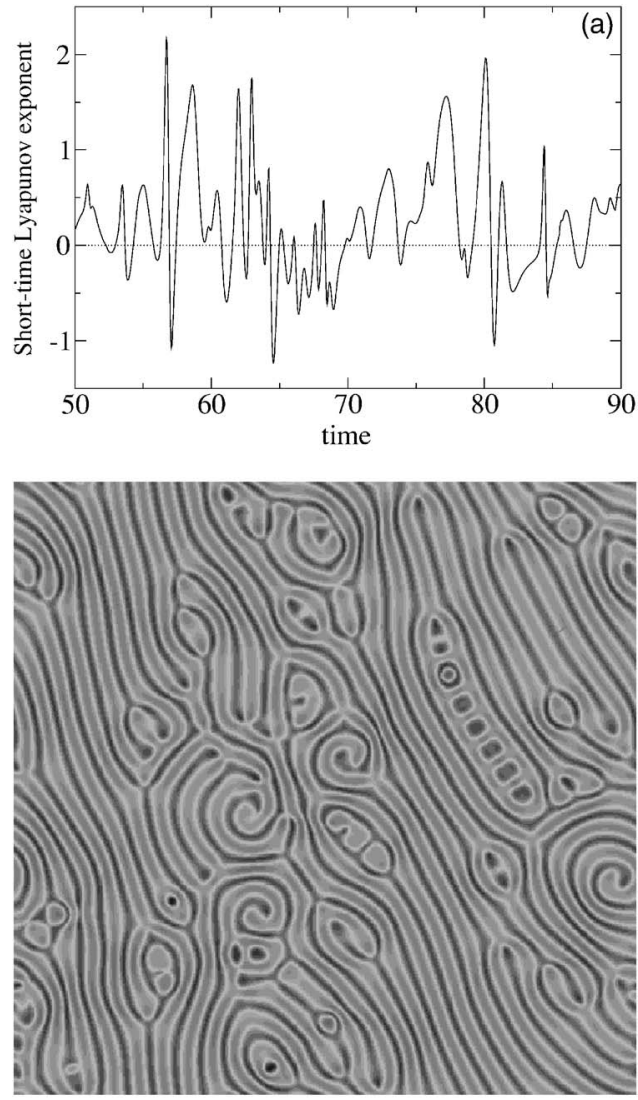

(b)

FIG. 5. (a) Evolution of the instantaneous largest Lyapunov exponent $\lambda_{1}^{\text {inst }}(t)$ for the spiral-defect chaotic state inside a periodic square cell of aspect ratio 64, for parameter values $R=3100, \sigma=1$, and $\Omega=0$. The average value of $\lambda_{1}^{\text {inst }}$ yields the estimate $\lambda_{1} \approx 0.18$, confirming the chaotic nature of this state. (b) The spatial pattern in the midplane $Z$-velocity field $u_{z}(t, x, y, z=1 / 2)$. Spiral defects are starting to form in the central third vertical region.

ture of the domain chaos state in rotating convecting cells. We also discuss how the largest Lyapunov exponent $\lambda_{1}$ scales near onset and how it varies with the aspect ratio $\Gamma$. The convection cells in all our simulations had no-slip walls with conducting lateral thermal boundary conditions.

\section{A. Relation of the exponent $\lambda_{1}^{\text {inst }}(t)$ to defect dynamics}

We carried out simulations on a rotating cylinder with aspect ratios in the range $10<\Gamma<40$, Prandtl number $\sigma$ $=0.93$, and angular speeds $\Omega=15$ and 17.6 , while varying the Rayleigh number over the range $2275<R<3000$. Linear theory predicts that the Küppers-Lortz instability [64] for rotating time-independent parallel nonlinear rolls occurs for rotation rates $\Omega_{c} \geqslant 13$ [65], so our values of $\Omega=15$ and 17.6 are slightly beyond the onset of the Küppers-Lortz instability. Figure 4 shows that the empirical critical number is $R_{c}$ $\approx 2117$ for the aspect ratio $\Gamma=20$.

For the reduced Rayleigh number $\epsilon=0.012$ [see Eq. (11)] and for a domain chaos state, Fig. 6 shows how $S_{\lambda}$ and hence the largest Lyapunov exponent $\lambda_{1}$ (whose value varies with the total integration time $T$ as it converges toward its infinite
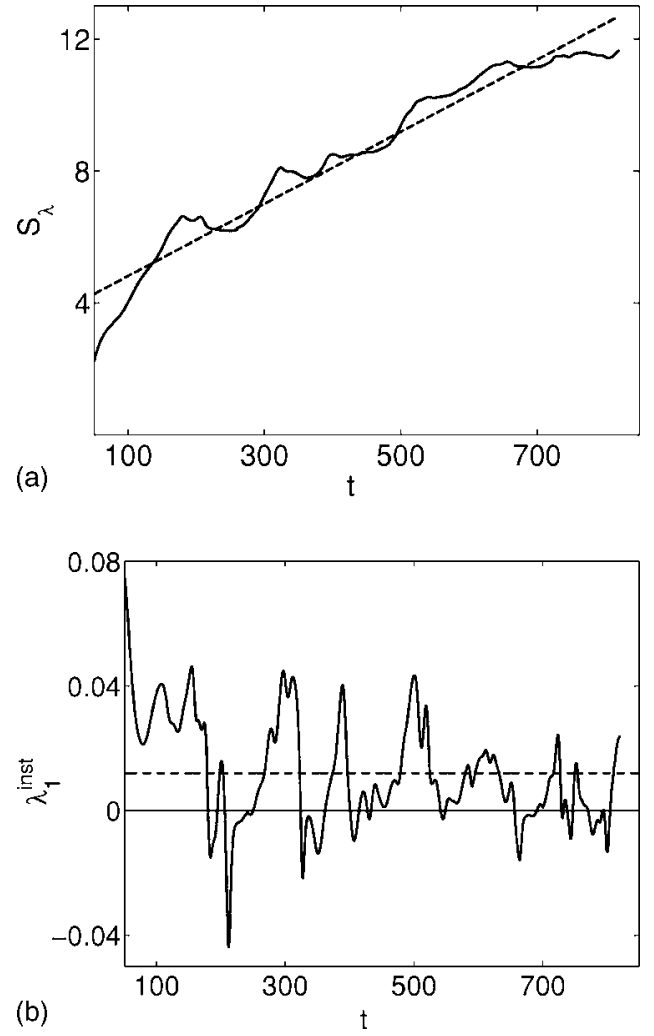

FIG. 6. (a) Evolution of $S_{\lambda}$ (6) as a function of time $t$, for a domain chaos state for the parameters $\Gamma=40, R=2275(\epsilon=0.012)$, $\sigma=0.93$, and $\Omega=17.6$. The convergence to the infinite time limit is slow and noisy. The slope of the dotted line gives a Lyapunov exponent of 0.011. (b) Corresponding instantaneous largest Lyapunov exponent $\lambda_{1}^{\text {inst }}(t)$ at time $t$ during the same time period as panel (a). The dashed line is the average value of $\lambda_{1}^{\text {inst }}(t)=0.012$, which is almost the same as $\lambda_{1}$.

time limit) as well as the instantaneous Lyapunov exponent $\lambda_{1}^{\text {inst }}(t)$, Eq. (3), change with time. Figure 6(a) shows that $\lambda_{1}$ converges slowly with large fluctuations toward its limit of $\lambda_{1} \approx 0.011$; this slow, noisy convergence is typical for highdimensional chaotic systems [compare Fig. 1(a)] and is one reason $\lambda_{1}$ can be difficult to estimate accurately. The corresponding instantaneous exponent in Fig. 6(b) looks similar to the curve obtained in Fig. 5(a) for spiral defect chaos. There are large, brief, positive fluctuations so that the overall dynamics is chaotic. There are also brief intervals when the quantity $\lambda_{1}^{\text {inst }}$ becomes negative, corresponding to temporarily increased stability of the dynamics.

The spatial structure that occurs close in time to the peaks of $\lambda_{1}^{\text {inst }}$ can be related to defect creation events. However, different defects have different effects on $\lambda_{1}^{\text {inst }}(t)$, and the situation is less clear than what was observed for spiral defect chaos [5]. Some defects have no effect at all on the leading $\lambda_{1}^{\text {inst }}(t)$, as can be seen by looking at the tangent space of the evolution.

Figure 7 shows the evolution of the $z$-component of the velocity field, $u_{z}(t, x, y, z=1 / 2)$, close in time to the peak in $\lambda_{1}^{\text {inst }}(t)$ that occurs at $t=110$ in Fig. 8 for the following parameters, $\Gamma=20, R=2800(\epsilon=0.33), \sigma=0.93$. The dynamics in the tangent space is visualized by plotting the linearized 

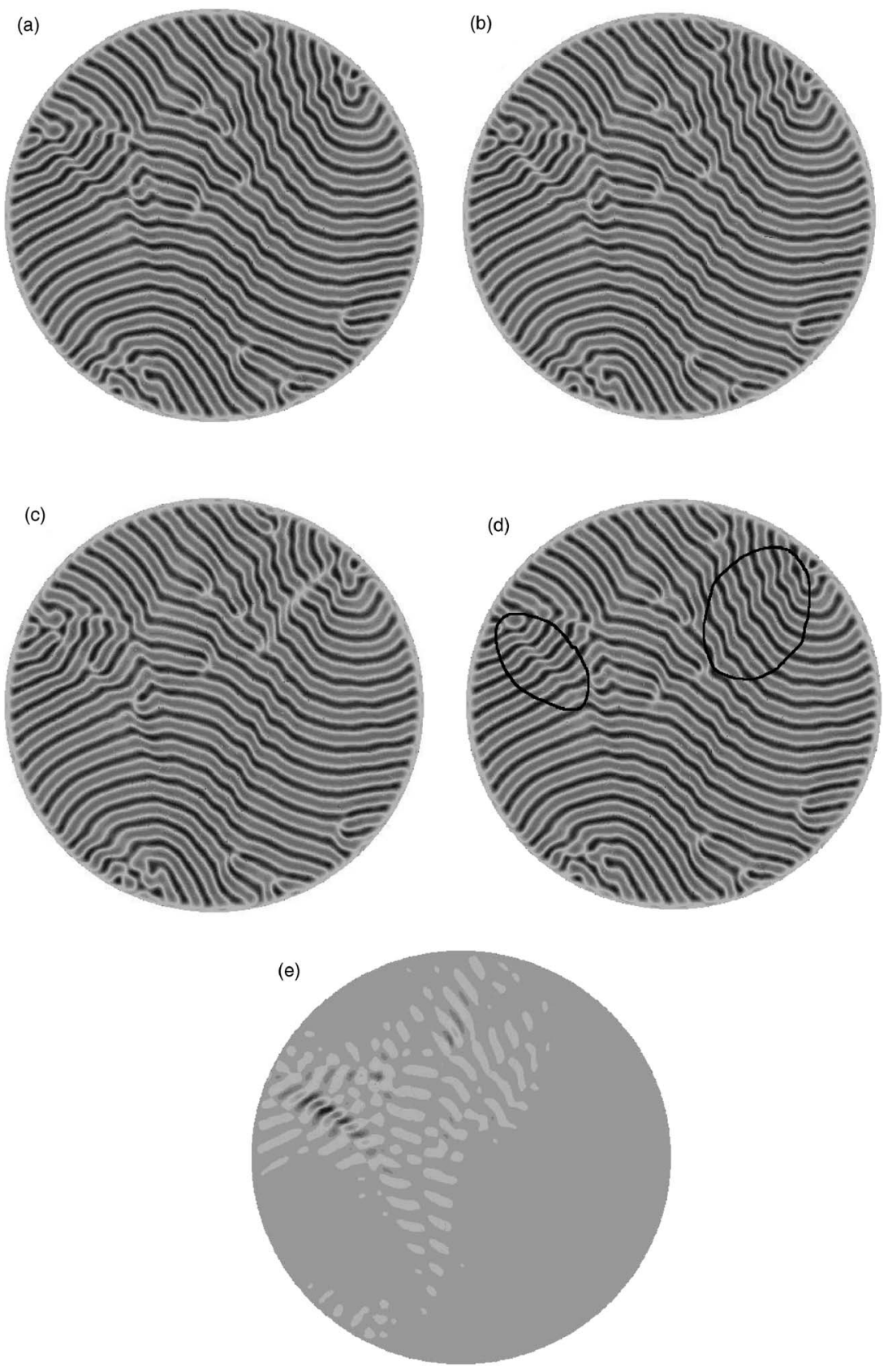

FIG. 7. (a) Evolution of the instantaneous largest Lyapunov eigenvector associated with $\lambda_{1}^{\text {inst }}$ for a domain chaos state in a rotating cylinder for parameters $\Gamma=20, \epsilon=0.33, \sigma=0.93$, and $\Omega=15$. Panels (a), (b), and (c) show the evolution of the midplane $z$ component of the velocity $u_{z}(t, x, y, 1 / 2)$ at times $t=109.59, t=110.15$, and $t=110.71$. This evolution occurs near the time at which there is a peak in the short-time Lyapunov exponent graph (Fig. 8). The panel (d) is a visual guide; it is a reproduction of panel (a) with the two black ellipses indicating where two defects occur. Panel (e) is a density plot of the linearized field $\delta u_{z}$, the tangent space for the field $u_{z}$ at time $t$ $=110.15$.

field $\delta u_{z}(t, x, y, z=1 / 2)$. Figure 7(e) shows this evolution. The lighter and darker regions indicate where the largest growth of the tangent space vectors occurs and so reveal the spatial structures that make the biggest contributions to the largest Lyapunov exponent.

The tangent space density plot [Fig. 7(e)] shows that the peak in the instantaneous exponent $\lambda_{1}^{\text {inst }}(t)$ at time $T$ $=110.15$ arises from the defect dynamics in the top left-hand corner of the cell. However, the defect structures developing in the top right-hand corner of the cell do not contribute substantially to the Lyapunov eigenvector. After studying numerous such spatial fields, we generally find that not all defect structures contribute equally to the eigenvector associated with the largest Lyapunov exponent. Possible reasons include the following: (1) the largest Lyapunov eigenvector is not along the instantaneous fastest stretching direction but has some memory of previous stretching; (2) in a large system, successive largest Lyapunov eigenvalues are close, and the defects that do not appear in the eigenvector spatial field associated with the largest exponent might be picked up by 


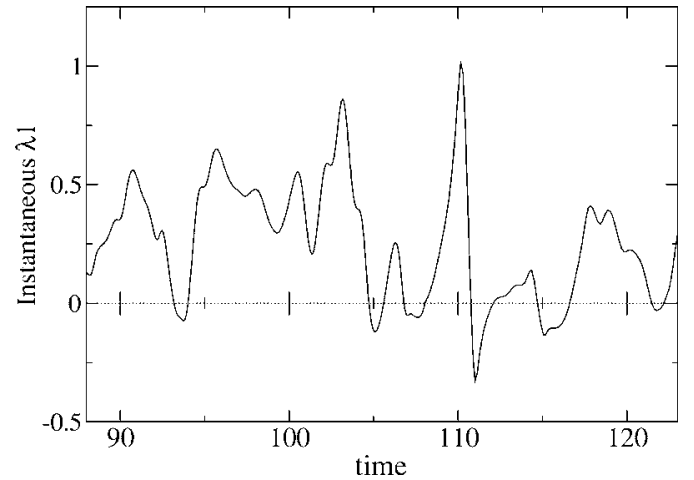

FIG. 8. Instantaneous largest Lyapunov exponent $\lambda_{1}^{\text {inst }}(t)$ as a function of time $t$, for a domain chaos state with $\Gamma=20, R=2800$ $(\epsilon=0.33), \sigma=0.93$. The corresponding spatial fields near the peak that occurs at $t=110$ are shown in Fig. 7.

the other eigenvectors; and (3) although at large enough times the tangent space vector is expected to converge to the eigenvector for the largest Lyapunov exponent, the time for this to happen is not known and may well be long in a large system. Hence, for a different set of perturbation initial conditions, we may find the defect on the top right-hand corner contributing more substantially. We plan on investigating these issues in more detail.

It is useful to contrast the behavior of Fig. 7(e) with results calculated by Chaté [36] of Lyapunov vectors associated with one-dimensional spatiotemporal intermittency. Chate found that the largest Lyapunov vector was highly spatially localized along turbulent patches [compare panels (a) and (b) of Fig. 1 of Ref. [36]], although a similar issue compared to our domain chaos calculation arises in that Chatés largest Lyapunov vector [his Fig. 1(b)] does not follow closely all the chaotic regions observed in his Fig. 1(a) [e.g., the chaotic patches on the far right-hand side of his Fig. 1(a) do not show up in his Fig. 1(b)] and we still do not understand under what conditions the spatial structure of the largest Lyapunov vector reflects the spatial structure of the chaotic state. Another difference is that the largest Lyapunov exponent $\lambda_{1}$ for one-dimensional (1D) spatiotemporal intermittency approaches a positive constant in the limit of approaching the onset of intermittency from above, while the exponent vanishes near the onset of domain chaos. Unlike spatiotemporal intermittency, domain chaos cannot be understood as a spatial union of laminar patches mixed with chaotic patches. Neither the extended defects (domain walls) nor the localized dislocations are intrinsically chaotic in their motion, it is their coupling to each other, and possibly driving by the lateral walls, that causes sustained chaotic dynamics. A more detailed study of the first 10 or more Lyapunov vectors along the lines of Ref. [36] and how they are localized would likely give a better insight into the domain chaos, but goes beyond the intent of this paper.

We also studied the correlations between $\lambda_{1}^{\text {inst }}$ and another diagnostic quantity, the reduced Nusselt number $N$. The Nusselt number is defined as the ratio of the total heat transported across the cell in the vertical direction divided by the heat transported via conduction only. Hence, if the Nusselt number is 1 , there is only conduction, and if the Nusselt
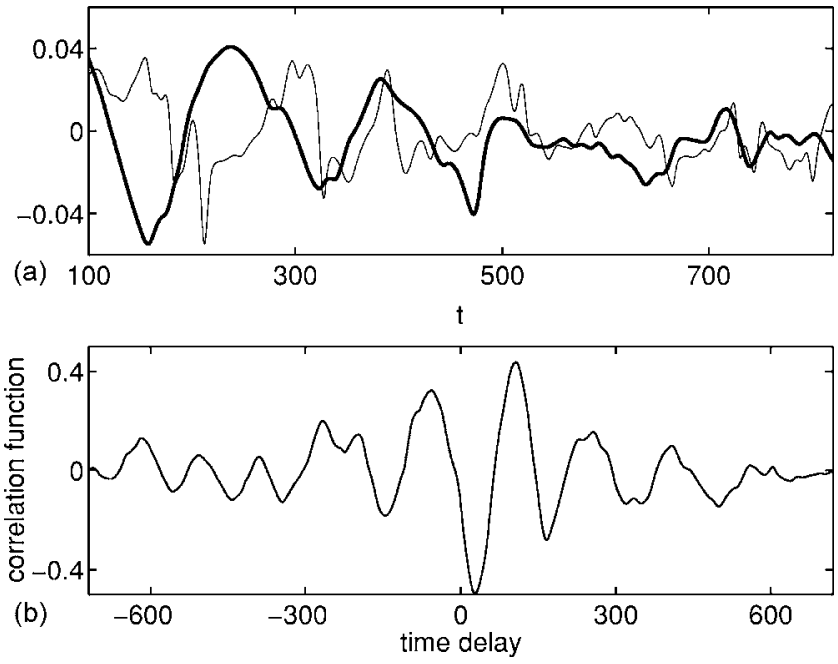

FIG. 9. (a) Reduced Nusselt number $N$ (thick curve) and instantaneous Lyapunov exponent $\lambda_{1}^{\text {inst }}$ (thin curve) as a function of time for the following parameters: $\Gamma=40, \sigma=0.93, \Omega=17.6, R=2275$ $(\epsilon=0.012)$. Note that the mean was subtracted from each of the dependent quantities, and their amplitudes have been rescaled for ease of comparison. (b) Correlation function between the reduced Nusselt number $N$ and the instantaneous Lyapunov exponent $\lambda_{1}^{\text {inst }}$.

number is greater than 1 , there is convection as well. To allow for a simpler comparison, we studied the reduced Nusselt number $N$; this is the Nusselt number -1 , which goes through zero at the onset of convection.

The reduced Nusselt number is a positive constant for a system of purely straight parallel rolls in its steady state, and it changes when the dynamics changes [66]. For example, when a dislocation is created, the quantity $N$ dips below its straight parallel roll value, since a system with a dislocation is a less efficient transporter of heat across the cell in the vertical direction. Conversely, if a dislocation is annihilated, $N$ will rise. Other changes, such as a local change in wave number or domain wall formation, will also cause changes in $N$. Since we are currently unable to use the Nusselt number to distinguish between dislocation creation/annihilation and other changes to the pattern, we will simply use the term "dynamical event" for any deviation from a straight parallel roll structure. Changes in $N$ correspond to dynamical events, and larger values of $N$ indicate regions of more order and vice versa.

We computed the correlation function between $N$ and $\lambda_{1}^{\text {inst }}$ as shown in Fig. 9 for $\Gamma=40, \sigma=0.93, \Omega=17.6$, and $R$ $=2275$. We see that $N$ is partially anticorrelated with $\lambda_{1}^{\text {inst: }}$ : The largest negative value in the correlation function occurs for close to zero time delay. We found this for other $R$ values as well.

It makes sense that $N$ is anticorrelated with $\lambda_{1}^{\text {inst }}$ because $\lambda_{1}^{\text {inst }}$ peaks when the system becomes more disordered, whereas an increase in disorder is associated with a dip in $N$. This anticorrelation indicates that dynamical events contribute to $\lambda_{1}^{\text {inst }}$, including but not exclusively, the dislocationnucleation events. This has also been seen in systems with smaller aspect ratio [67]. We note that while the value of the anticorrelation near zero lag in Fig. 9 is significant, it is not 

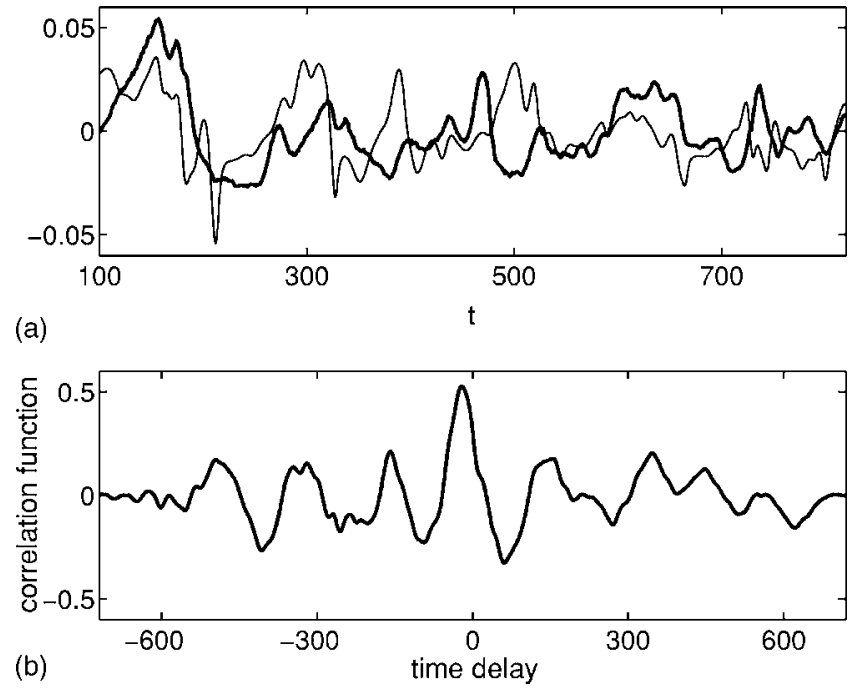

FIG. 10. (a) Defect density $\rho_{d}$ (thick curve) and instantaneous Lyapunov exponent $\lambda_{1}^{\text {inst }}$ (thin curve) as a function of time for the following parameters: $\Gamma=40, \sigma=0.93, \Omega=17.6, \quad R=2275 \quad(\epsilon$ $=0.012$ ). Note that the mean was subtracted from each of the dependent quantities, and their amplitudes were independently rescaled for ease of comparison. (b) Correlation function between the defect density $\rho_{d}$ and the instantaneous Lyapunov exponent $\lambda_{1}^{\text {inst }}$.

perfect, indicating that other factors may contribute to changes in $\lambda_{1}^{\text {inst }}$ that cannot be detected by looking at $N$.

Because the Nusselt number $N$ is anticorrelated with $\lambda_{1}^{\text {inst }}$ and $N$ is itself related to the pattern of defects, we also studied directly the correlation between $\lambda_{1}^{\text {inst }}$ and the defect density $\rho_{d}$ for the parameter values $\Gamma=40, \sigma=0.93, \Omega=17.6$, and $R=2275$. We computed an area of the flow associated with defects as described in Ref. [68], by identifying regions of large local curvature in the computed local unit wave vector. The defect density $\rho_{d}$ was then calculated as the ratio of the total area of defects to the total area of the cell. We then computed the correlation function between $\rho_{d}$ and $\lambda_{1}^{\text {inst }}$ as shown in Fig. 10. We see that $\rho_{d}$ is partially correlated with $\lambda_{1}^{\text {inst }}$ since there is a relatively large peak near zero time delay. However, the correlation is not perfect. Figure 10(b) provides quantitative evidence of our previously qualitative observation that some, but not all, defects contribute to $\lambda_{1}^{\text {inst }}$. Just as with the Nusselt number $N$, our method of counting defects is unable to distinguish between dislocations and domain walls, so we were unable to compare and contrast the correlations between $\lambda_{1}^{\text {inst }}$ and specific types of defects.

\section{B. Scaling of the largest Lyapunov exponent $\lambda_{1}$ near onset}

The domain chaos state in the rotating convection system emerges continuously from a homogeneous state. To the extent that the dynamics is described by the amplitude equation formalism, we then expect the Lyapunov exponent $\lambda_{1}$ to go continuously to zero as onset is approached. The amplitude equation formalism predicts that, sufficiently close to onset, all time scales and so $\lambda_{1}$ should scale with $\epsilon$ with a scaling exponent of $-1[27,30]$. In this section, we test this prediction using our spectral element code to simulate large rotating convecting flows near onset.

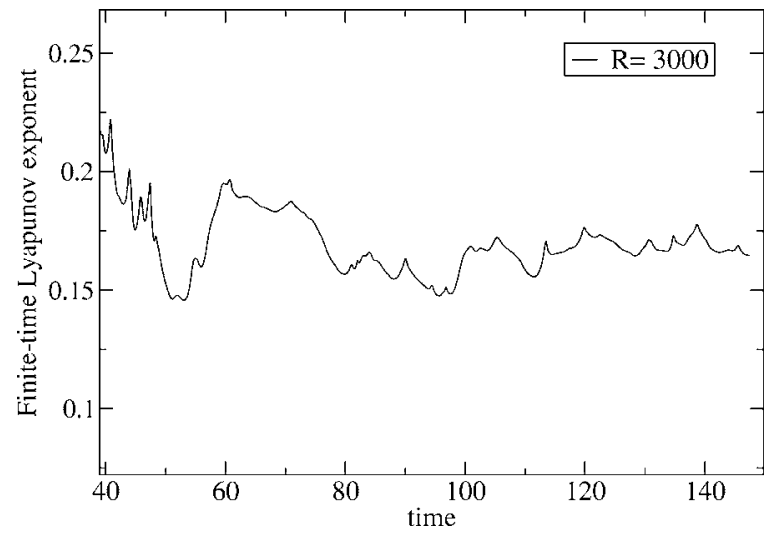

FIG. 11. Convergence of the largest Lyapunov exponent $\lambda_{1}(t)$ toward its asymptotic value for an aspect ratio $\Gamma=10$ system $(\epsilon$ $=0.39, \Omega=15$, and $\sigma=0.93$ ).

A serious problem when computing Lyapunov exponents, especially for systems with many degrees of freedom, is that the convergence is slow. It has been conjectured that these systems converge to their asymptotic values as $t^{-\alpha}$, and numerical experiments in finite-dimensional systems have found values of $\alpha$ between 0.5 and 1 depending on the nature of the system [51]. Typical convergence behavior of the leading Lyapunov exponent in the $\Gamma=10$ system is shown in Fig. 11. The convergence is slower for $\Gamma=20$ and 40. As we approach onset, it could take up to 500 to 1000 vertical diffusion times to obtain a reliable value for $\lambda_{1}$ leading to costly calculations. To overcome this issue, we computed $\lambda_{1}$ for $\Gamma=20$ systems by running three separate simulations with different initial conditions and then averaging the Lyapunov exponent estimates from those simulations. For the $\Gamma=40$ case, we simply ran each simulation out to at least 800 vertical diffusion times.

Figure 12 shows that $\lambda_{1}$ approaches zero linearly as we approach onset for each aspect ratio. We note that the best-fit line shows that $\lambda_{1}$ reaches zero at a nonzero value of $\epsilon$, which decreases with aspect ratio. The $x$ intercept is 0.03 for $\Gamma=10,20$ and decreases to 0.007 for $\Gamma=40$. This indicates that the linear scaling is not valid close to onset for smaller aspect ratios.

We performed a linear fit to the data from Fig. 12(b) on a $\log$-log plot. The slope of this line also gives us the scaling with $\epsilon$. We found the slope to be 1.1, in good agreement with a linear dependence of $\lambda_{1}$ on $\epsilon$.

\section{The dependence of the Lyapunov exponent $\lambda_{1}$ on aspect ratio}

The rigid lateral walls of the convection cell impose strong restrictions on the dynamics inside the cell, since the fluid velocity is forced to be zero at the cell wall. Another related effect of the rigid boundary is that the rolls are forced to intersect the wall transversely. This restriction implies that the strength of chaos in the cell depends on the surface to volume ratio for moderate values of $\Gamma$. Figure 13 confirms this dependence. For a given $\epsilon$, the dynamics in the larger aspect ratio cell has higher value of $\lambda_{1}$. One would expect 

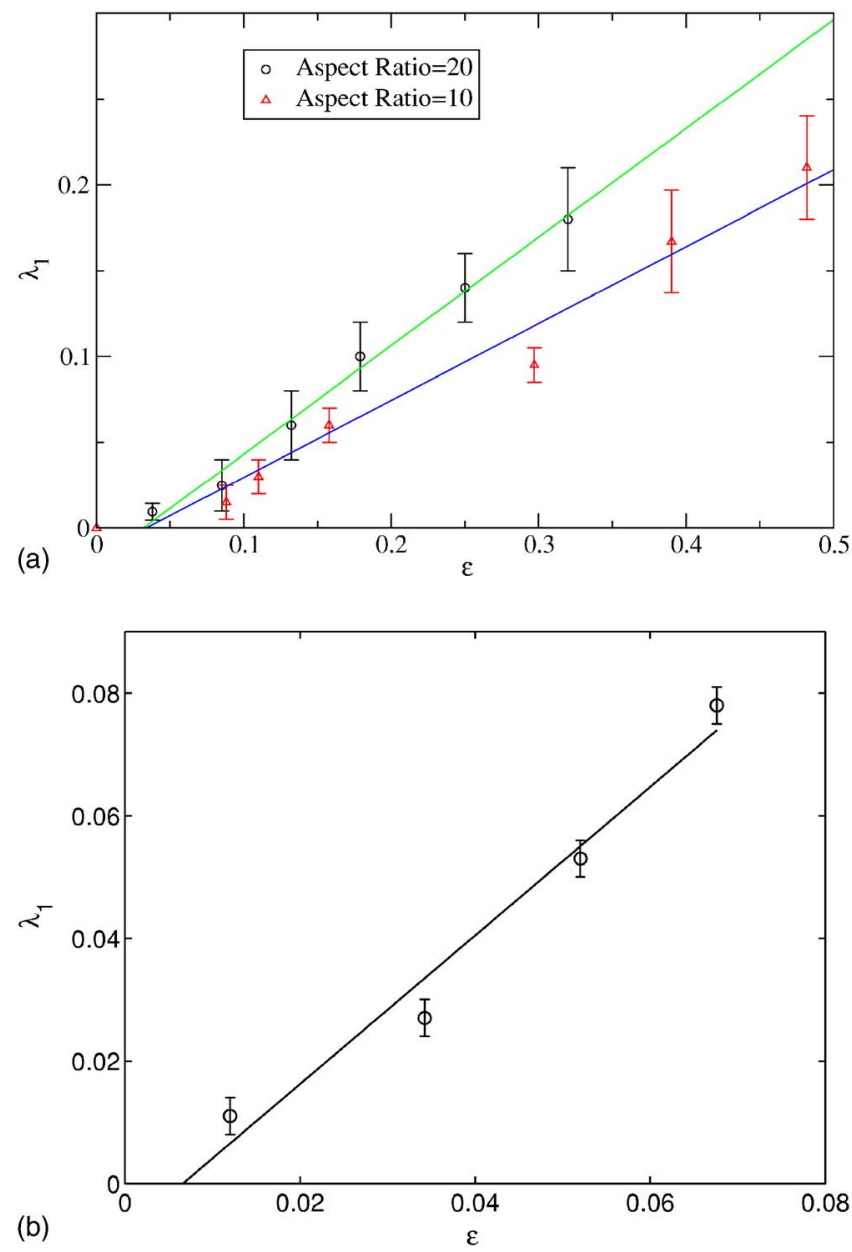

FIG. 12. (Color online) Largest Lyapunov exponent $\lambda_{1}$ vs the reduced Rayleigh number $\epsilon$ for aspect ratios $\Gamma=10$ and 20 in (a) and aspect ratio $\Gamma=40$ in (b). The solid lines are linear-least-squares fits to calculated points and intercept the $\epsilon$ axis at the value $\epsilon$ $=0.03$ for (a) and $\epsilon=0.007$ for (b). The slopes of the fitted lines are $0.45,0.63$, and 1.21 for $\Gamma=10,20$, and 40 , respectively. The amplitude-equation formalism predicts a linear scaling for the various time scales with respect to $\epsilon$ [26].

that for very large aspect ratio cells the boundary effects would be minimal and hence $\lambda_{1}$ would not show an aspect ratio dependence. We do not see any flattening of the curve for $20<\Gamma<40$. We note that this aspect ratio dependence does appear to flatten out as $\Gamma$ becomes smaller (compare $\Gamma=20$ to $\Gamma=10$ ). For small aspect ratio systems, the boundary more completely dominates the dynamics.

Given that the dependence of $\lambda_{1}$ in Fig. 13 is contrary to that expected of an intensive quantity in a large-aspect-ratio cell, we also examined how the standard deviation $\sigma$ (rootmean-square time average) of $\lambda_{1}^{\text {inst }}$ depended on $\Gamma$. This too had a surprising dependence as shown in Fig. 14, in that $\sigma$ decreases with increasing aspect ratio. This indicates that the fluctuations in $\lambda_{1}^{\text {inst }}$ get smaller with aspect ratio.

\section{CONCLUSIONS}

For the first time, we have computed the largest Lyapunov exponent $\lambda_{1}$ and its behavior near onset for the domain chaos

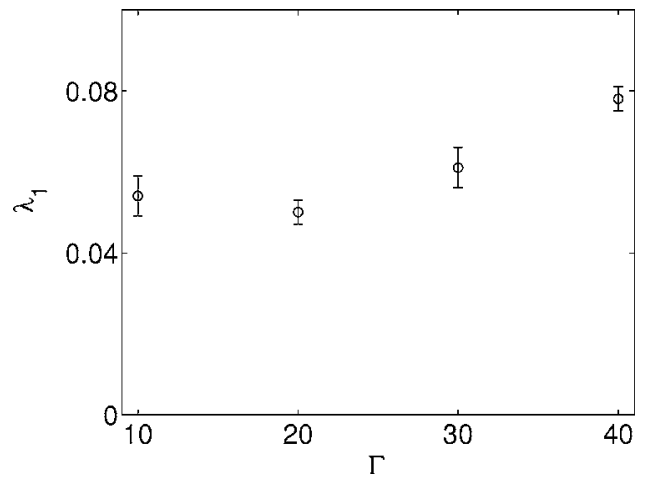

FIG. 13. The largest Lyapunov exponent $\lambda_{1}$ versus the aspect ratio $\Gamma$ for the domain chaos state of a convecting fluid in a rotating cylindrical cell with conducting lateral walls. The approximately linear dependence (for $20<\Gamma<40$ ) shows that the exponent $\lambda_{1}$ does not act as an intensive quantity, which would be expected for localized sources of chaos. For these runs, the Rayleigh number, rotation rate, and Prandtl number had the values $R=2400$ ( $\epsilon$ $=0.064), \Omega=17.6$, and $\sigma=0.93$.

state of a convecting fluid with experimentally realistic boundary conditions. We have shown that $\lambda_{1}$ scales linearly with respect to $\epsilon$, in agreement with the predictions of amplitude equation theory that time scales near onset should diverge as $\epsilon^{-1}$. This result is in disagreement with experiments, which find a smaller exponent. We do, however, find that the linear scaling of $\lambda_{1}$ does not extend all the way to onset for smaller aspect ratio [as the nonzero intercept in Fig. 12(a) indicates].

In Ref. [39], Egolf suggested that the value of $\lambda_{1}$ for the SDC state was determined primarily by short-lived, spatially localized dislocation nucleation events. In this paper we have shown that although there is a correlation between defect generation and contributions to $\lambda_{1}$, not all defects contribute to the leading Lyapunov exponent. It is, however, possible that some of the defects that do not contribute to the leading exponent could contribute to the higher-order Lyapunov exponents. We also have seen that the reduced Nusselt number $N$ is somewhat anticorrelated with $\lambda_{1}^{\text {inst }}$, indicating that both quantities are a good measure of the occurrence of dynamical events in the system.

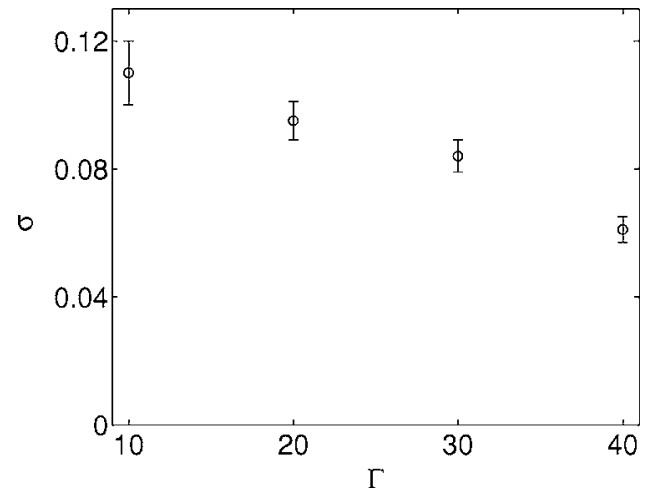

FIG. 14. Plot of the root-mean-square time average $\sigma$ of the instantaneous Lyapunov exponent $\lambda_{1}^{\text {inst }}$ versus the aspect ratio $\Gamma$ for the same parameters as in Fig. 13. The fluctuations about $\lambda_{1}$ decrease with $\Gamma$. 
Computations of $\lambda_{1}$ in some of the mathematical model systems $[36,38-40,69]$ show that $\lambda_{1}$ is largely independent of system size. Egolf's calculation for the SDC state (with periodic boundary conditions) also shows that system size does not change $\lambda_{1}$. Our computations of the SDC state confirm Egolf's results. For the domain chaos state, however, we find an unexpected nonintensive behavior of $\lambda_{1}$ for $20 \leqslant \Gamma$ $\leqslant 40$. The expectation of intensive behavior of $\lambda_{1}$ comes from the arguments originally given by Ruelle [70] that for large systems distant regions will be dynamically independent. The observed nonintensive behavior is possibly a consequence of the extended fronts, which can grow in size with increasing $\Gamma$. This nonintensive behavior of $\lambda_{1}$ might therefore imply that for the domain chaos state, long-range correlations affect the dynamics in distant regions [27]. Computational experiments in larger domains (which are currently too expensive) are required to understand this phenomenon. Since SDC has a $\lambda_{1}$ independent of aspect ratio and since domain chaos shows an aspect ratio dependence, it would also be interesting to study the transition as a function of rotation rate from SDC to domain chaos.

We have emphasized the local properties $\lambda_{1}^{\text {inst }}$ of the largest Lyapunov exponent $\lambda_{1}$ in this paper. Egolf has speculated [39] that the Kaplan-Yorke conjecture that relates the spectrum of exponents $\lambda_{i}$ to the Lyapunov fractal dimension $D_{\mathcal{L}}$ may also hold locally in time. One can then estimate local exponents $\lambda_{i}^{\text {inst }}$ and a local (in time) fractal dimension $D^{\text {inst }}(t)$. It would be interesting to calculate this local dimension for representative physical examples of spatiotemporal chaos such as spiral defect chaos and domain chaos.

\section{ACKNOWLEDGMENTS}

The authors thank Michael Cross and Mark Paul for helpful discussions. This work was supported by DOE Grants Nos. DE-FG02-98ER14892, DE-FG03-98ER14891, and DEFG02-98ER14892; and by the Mathematical, Information, and Computational Sciences Division subprogram of the Office of Advanced Scientific Computing Research, U.S. Department of Energy, under Contract No. W-31-109-Eng-38. The numerical code was run on the following supercomputing sites, whom the authors gratefully acknowledge: the National Computational Science Alliance under DMR040001, which utilized the NCSA Xeon Linux Supercluster; the National Energy Research Scientific Computing Center, which is supported by the Office of Science of the U.S. Department of Energy under Contract No. DE-AC03-76SF00098; and "Jazz," a 350-node computing cluster operated by the Mathematics and Computer Science Division at Argonne National Laboratory as part of its Laboratory Computing Resource Center.
[1] M. C. Cross and P. C. Hohenberg, Rev. Mod. Phys. 65, 851 (1993).

[2] M. C. Cross and P. C. Hohenberg, Science 263, 1569 (1994).

[3] H. S. Greenside, in Semi-Analytic Methods for the Navier Stokes Equations, edited by K. Coughlin (American Mathematical Society, Providence, RI, 1999), Vol. 20 of CRM Proceedings and Lecture Notes, pp. 9-40, e-print chao-dyn/ 9612004

[4] K.-H. Chiam, M. C. Cross, H. S. Greenside, and P. F. Fischer, Phys. Rev. E 71, 036205 (2005).

[5] D. A. Egolf, I. V. Melnikov, W. Pesch, and R. E. Ecke, Nature (London) 404, 733 (2000).

[6] S. W. Morris, E. Bodenschatz, D. S. Cannell, and G. Ahlers, Phys. Rev. Lett. 71, 2026 (1993).

[7] M. Assenheimer and V. Steinberg, Nature (London) 367, 345 (1994).

[8] Y. Hu, R. Ecke, and G. Ahlers, Phys. Rev. E 51, 3263 (1995).

[9] R. E. Ecke, Y. Hu, R. Mainieri, and G. Ahlers, Science 269, 1704 (1995).

[10] J. Liu and G. Ahlers, Phys. Rev. Lett. 77, 3126 (1996).

[11] S. Morris, E. Bodenschatz, D. Cannell, and G. Ahlers, Physica D 97, 164 (1996).

[12] R. E. Ecke, Physica A 239, 174 (1997).

[13] H.-W. Xi, X.-J. Li, and J. D. Gunton, Phys. Rev. Lett. 78, 1046 (1997).

[14] M. Cross, Physica D 97, 65 (1996).

[15] X.-J. Li, H.-W. Xi, and J. D. Gunton, Phys. Rev. E 57, 1705 (1997).

[16] W. Decker, W. Pesch, and A. Weber, Phys. Rev. Lett. 73, 648
(1994).

[17] D. A. Egolf, I. V. Melnikov, and E. Bodenschatz, Phys. Rev. Lett. 80, 3228 (1998).

[18] S. M. Zoldi, J. Liu, K. M. S. Bejaj, H. S. Greenside, and G. Ahlers, Phys. Rev. E 58, R6903 (1998).

[19] R. Schmitz, W. Pesch, and W. Zimmermann, Phys. Rev. E 65, 037302 (2002).

[20] K.-H. Chiam, M. R. Paul, M. C. Cross, and H. S. Greenside, Phys. Rev. E 67, 056206 (2003).

[21] Y. Hu, R. E. Ecke, and G. Ahlers, Phys. Rev. Lett. 74, 5040 (1995).

[22] Y. Hu, W. Pesch, G. Ahlers, and R. E. Ecke, Phys. Rev. E 58, 5821 (1998).

[23] L. Ning and R. E. Ecke, Phys. Rev. E 47, 3326 (1993).

[24] F. Zhong, R. Ecke, and V. Steinberg, Physica D 51, 596 (1991).

[25] K. M. S. Bejaj, G. Ahlers, and W. Pesch, Phys. Rev. E 65, 056309 (2002).

[26] Y. Tu and M. C. Cross, Phys. Rev. Lett. 69, 2515 (1992).

[27] M. C. Cross, D. Meiron, and Y. Tu, Chaos 4, 607 (1994).

[28] Y. Ponty, T. Passot, and P. L. Sulem, Phys. Rev. E 56, 4162 (1997).

[29] D. Laveder, T. Passot, Y. Ponty, and P. L. Sulem, Phys. Rev. E 59, R4745 (1999).

[30] M. C. Cross, M. Louie, and D. Meiron, Phys. Rev. E 63, 045201(R) (2001).

[31] J. D. Scheel and M. C. Cross, Phys. Rev. E 72, 056315 (2005).

[32] D. R. Rasmussen and T. Bohr, Phys. Lett. A 125, 107 (1987).

[33] E. J. Ding and Y. N. lu, Phys. Lett. A 161, 357 (1992). 
[34] R. Livi, A. Politi, and S. Ruffo, J. Phys. A 25, 4813 (1992).

[35] F. Kaspar and H. G. Schuster, Phys. Lett. 113A, 451 (1986).

[36] H. Chaté, Europhys. Lett. 21, 419 (1993).

[37] D. A. Egolf and H. S. Greenside, Nature (London) 369, 129 (1994).

[38] C. S. O'Hern, D. A. Egolf, and H. S. Greenside, Phys. Rev. E 53, 3374 (1996).

[39] D. A. Egolf, Phys. Rev. Lett. 81, 4120 (1998).

[40] M. C. Strain and H. S. Greenside, Phys. Rev. Lett. 80, 2306 (1998)

[41] E. Ott, Chaos in Dynamical Systems, 2nd ed. (Cambridge University Press, New York, 2002).

[42] H. Kantz and T. Schreiber, Nonlinear Time Series Analysis, 2nd ed. (Cambridge University Press, New York, 2003).

[43] E. Aurell, G. Boffetta, A. Crisanti, G. Paladin, and A. Vulpiani, Phys. Rev. E 53, 2337 (1996).

[44] T. S. Parker and L. O. Chua, Practical Numerical Algorithms for Chaotic Systems (Springer-Verlag, New York, 1989).

[45] S. M. Zoldi and H. S. Greenside, Phys. Rev. Lett. 78, 1687 (1997).

[46] J. A. Vastano and R. D. Moser, J. Fluid Mech. 233, 83 (1991).

[47] J.-P. Eckmann and I. Procaccia, Phys. Rev. A 34, 659 (1986).

[48] H. D. Abarbanel, R. Brown, and L. S. Tsimring, Rev. Mod. Phys. 65, 1331 (1993).

[49] A. Prasad and R. Ramaswamy, Phys. Rev. E 60, 2761 (1999).

[50] B. Eckhardt and D. Yao, Physica D 65, 100 (1993).

[51] H. D. I. Abarbanel, N. Brown, and M. B. Kennel, J. Nonlinear Sci. 1, 175 (1991).

[52] F. H. Busse, Rep. Prog. Phys. 41, 1929 (1978).

[53] G. Ahlers (private communication).
[54] H. M. Tufo and P. F. Fischer, Proceedings of the ACM/IEEE SC99 Conference on high performance networking and computing, IEEE Computer Society (CD-ROM format, 1999), Vol. SC99, ftp://info.mcs.anl.gov/pub/tech_reports/reports/ P762.ps.Z

[55] P. F. Fischer, J. Comput. Phys. 133, 84 (1997).

[56] M. R. Paul, K. H. Chiam, M. C. Cross, P. F. Fischer, and H. S. Greenside, Physica D 184, 114 (2003).

[57] A. Jayaraman and H. S. Greenside (in preparation).

[58] M. R. Paul, K.-H. Chiam, M. C. Cross, P. F. Fischer, and H. S. Greenside, Physica D 184, 114 (2003).

[59] M. R. Paul, M. C. Cross, and P. F. Fischer, Phys. Rev. E 66, 046210 (2002).

[60] J. D. Scheel, M. R. Paul, M. C. Cross, and P. F. Fischer, Phys. Rev. E 68, 066216 (2003).

[61] S. Chandrasekhar, Hydrodynamic and Hydromagnetic Stability (Clarendon, Oxford, 1968).

[62] M. C. Cross, Phys. Fluids 23, 1727 (1980).

[63] D. A. Egolf (private communication).

[64] G. Küppers and D. Lortz, J. Fluid Mech. 35, 609 (1969).

[65] E. Bodenschatz, W. Pesch, and G. Ahlers, Annu. Rev. Fluid Mech. 32, 709 (2000).

[66] M. R. Paul, M. C. Cross, P. F. Fischer, and H. S. Greenside, Phys. Rev. Lett. 87, 154501 (2001).

[67] J. D. Scheel, Ph.D. thesis, California Institute of Technology, 2006.

[68] M. R. Paul, K.-H. Chiam, M. C. Cross, and P. F. Fischer, Phys. Rev. Lett. 93, 064503 (2004).

[69] F. Sastre and G. Pérez, Phys. Rev. E 57, 5213 (1998).

[70] D. Ruelle, Commun. Math. Phys. 87, 287 (1982). 